\title{
Single feed circularly polarized crescent-cut and extended corner square microstrip antennas for wireless biotelemetry
}

\author{
Mehdi Hasan Chowdhury ${ }^{1}$, Quazi Delwar Hossain ${ }^{2}$, Md. Azad Hossain ${ }^{3}$, Ray Chak Chung Cheung ${ }^{4}$ \\ ${ }^{1,4}$ Department of Electronic Engineering, City University of Hong Kong, Hong Kong SAR \\ ${ }^{2}$ Department of Electrical \& Electronic Engineering, Chittagong University of Engineering \& Technology, Bangladesh \\ ${ }^{3}$ Department of Electronics \& Telecommunication Engineering, Chittagong University of Engineering \& Technology, \\ Bangladesh
}

\begin{tabular}{|c|c|}
\hline Article Info & ABSTRACT \\
\hline $\begin{array}{l}\text { Article history: } \\
\text { Received May 9, } 2018 \\
\text { Revised Oct 22, } 2018 \\
\text { Accepted Dec 3, } 2018\end{array}$ & $\begin{array}{l}\text { In this paper, the development of two novel circularly polarized microstrip antennas is } \\
\text { thoroughly explained. These antennas are fed by coaxial feeding technique. One of the } \\
\text { primary objectives of the proposed work is to tune the antennas to work in ISM band. } \\
\text { This frequency band refers to the internationally recognized radio frequency band- } \\
\text { width which is to be used explicitly for Industrial, Scientific, and Medical applications. }\end{array}$ \\
\hline $\begin{array}{l}\text { Keywords: } \\
\text { Circular polarization } \\
\text { Microstrip antenna } \\
\text { Single-feed } \\
\text { ISM band } \\
\text { Wireless biotelemetry }\end{array}$ & $\begin{array}{l}\text { Therefore, these antennas would be suitable to use in the field of wireless biotelemetry. } \\
\text { Two new antenna design techniques have been introduced to produce circular polar- } \\
\text { ization, and details of these schemes are described. The proposed microstrip antennas } \\
\text { are designed and simulated on Advanced Design System (ADS) software. The return } \\
\text { loss of the proposed crescent-cut antenna is }-19.3 \mathrm{~dB} \text { at the operating frequency. The } \\
\text { extended corner antenna has the return loss of }-29.3 \mathrm{~dB} \text { at the tuned frequency. The } \\
\text { simulation results are also presented and discussed. }\end{array}$ \\
\hline
\end{tabular}

\section{Corresponding Author:}

Mehdi Hasan Chowdhury,

Department of Electronic Engineering,

City University of Hong Kong,

Kowloon, Hong Kong SAR.

Email: mhchowdhury.eee@gmail.com

\section{INTRODUCTION}

Telemetry means accumulating data from a remote location and then forwarding the information to a suitable place to be examined and documented. Wireless biotelemetry is the distinguished field of biomedical instrumentation that enables transmission and reception of bio-signals from an inconvenient location to a convenient remote monitoring site without using wires [1]. Meticulous research activities in the field of biotelemetry have been carried out in recent years due to its significant impact on patient monitoring in distant non-clinical environments, such as old communities, post-surgery patients at homes, military bases, etc. [2]. Observing a patient at home for an extended duration can help a physician to follow disease progression. Thus, the earlier treatment can save many lives, and it will be only achievable if detection of a disease can be done timely.

However, telecommunication frequency bands are regulated by the Federal Communications Commission (FCC) and similar agencies [3]. Frequency band $2.4 \mathrm{GHz}$ to $2.5 \mathrm{GHz}$ is dedicated for the ISM band (industrial, scientific and medical radio band)[4], which belongs to the internationally reserved radio spectrum for the scientific, medical and industrial applications [5]. This frequency bandwidth can be used in numerous applications, including the Wireless Biotelemetry, without a license in most countries [6].

The size, weight, cost, and performance of the antennas in the field of wireless biotelemetry are constraints and to meet these requirements, microstrip antennas can be used as they offer simple structure, lowcost small size, and high polarization [7]. A microstrip antenna is manufactured using microstrip techniques 
on a PCB (Printed Circuit Board) and frequently used in microwave frequency communication.

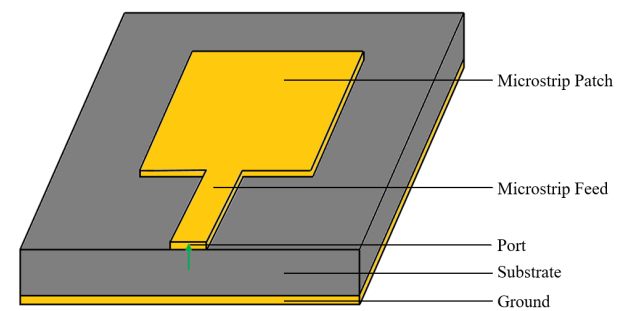

Figure 1. Basic Structure of a microstrip patch antenna [8]

If we consider a simple microstrip antenna, as shown in the Figure 1, it consists of a pair of parallel conducting layers separated by a dielectric substrate. The upper conducting layer (also known as the 'Patch') is the source of radiation, and the lower layer is the ground plane. Typically microstrip patch antennas are rectangular or circular, but it is possible to make them in any reasonable geometrical shape.

Polarization is one of the most vital factors in wave propagation between the transmitting and receiver antennas. The polarization of an antenna in a particular direction defines as the polarization of the wave transmitted by the antenna'. There are three types of polarization: linear polarization, elliptical polarization, and circular polarization [9]. An antenna is linearly polarized if the field vector (electric or magnetic) for the transmitted wave of the antenna only has one component or two orthogonal linear component that are in time phase or $180^{\circ}$ (or multiples of $180^{\circ}$ ) out of phase.

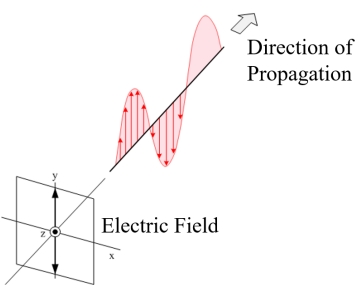

(a) Linear Polarization

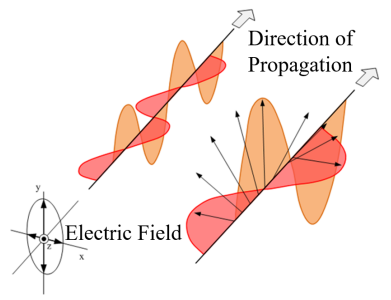

(b) Elliptical Polarization

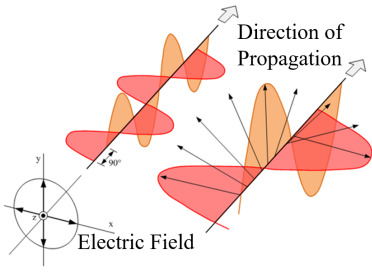

(c) Circular Polarization

Figure 2. Different polarization of antenna [10]

An antenna radiates elliptically polarized wave when the field vector (electric or magnetic) for the transmitted wave of the antenna has two unequal orthogonal linear components and the phase difference between them is odd multiples of $90^{\circ}$. When the field vector (electric or magnetic) for the transmitted wave of the antenna has two orthogonal linear components with the same magnitude and the phase difference between them is odd multiples of $90^{\circ}$, then antenna has the circular polarization (CP). Figure 2 shows how different polarized wave propagates.

CP offers multiple benefits over linear polarization [10]. If linearly polarized antennas are used, both the receiving and transmitting antenna must be aligned to avoid polarization mismatch. For circular polarization, this is not essential. Additionally, CP antennas are immune to Faraday rotation, and they can mitigate multipath fading. Therefore, $\mathrm{CP}$ antennas offer the benefit of high data rate communication, as it decreases interference between direct and reflected signal.

\section{STATE OF THE ART}

Single probe-fed circularly polarized microstrip antennas are highly helpful in situations where it is difficult to implement dual-orthogonal feeds. Many designs of circularly polarized single-feed microstrip antennas with square or circular patches are known. Usually, a microstrip patch with a single-point feed emits linear polarization. If circular polarization is needed, it will be required for two orthogonal patch modes with the same amplitude and with $90^{\circ}$ phase difference to be induced. 


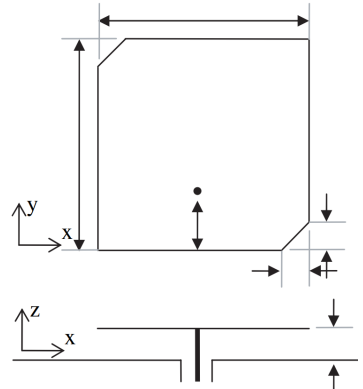

(a) Single probe-fed circularly polarized truncated corner microstrip antenna [11]

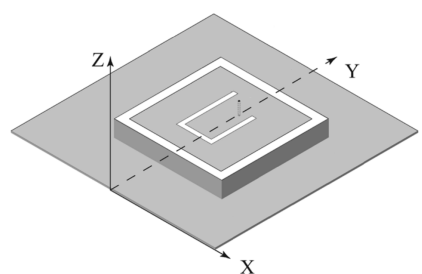

(b) Single probe-fed circularly polarized U-slot patch antenna [12]

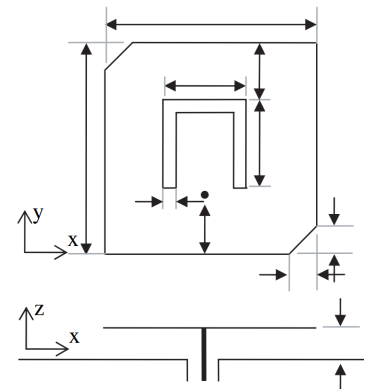

(c) Single probe-fed circularly polarized truncated corner microstrip antenna with U-slot [11]

Figure 3. Some common configuration of single probe-fed circularly polarized rectangular microstrip antenna

This technique can be implemented by slightly perturbing a microstrip antenna patch at proper places. Conventionally this is done by truncating the corners of a rectangular microstrip patch (Figure 3a) [11] or introducing slots inside the radiating patch (Figure 3b) [12].

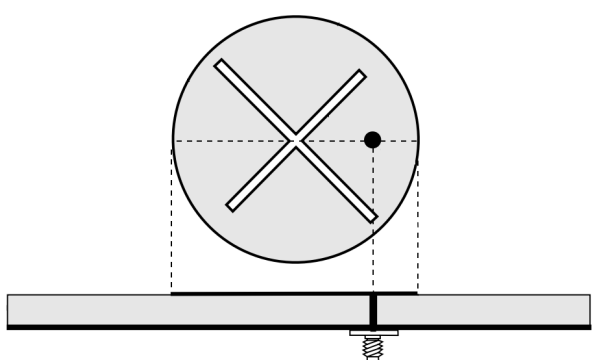

(a) Single probe-fed circularly polarized circular microstrip antenna with a cross-slot of unequal arm lengths [13]

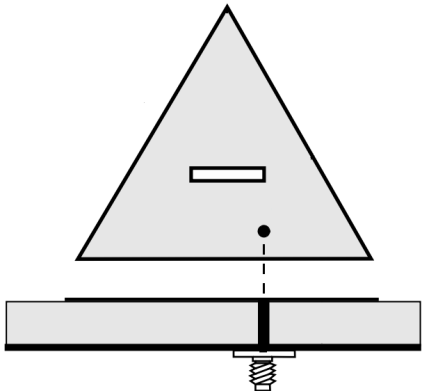

(b) Single probe-fed circularly polarized triangular microstrip antennas with a horizontal narrow slot [14]

Figure 4. Some common configuration of single probe-fed circularly polarized circular and triangular microstrip antenna

Sometimes these two techniques are combined to assure better performance (Figure 3c) [11]. However, It is also possible to design circular (Figure 4a) [13], triangular (Figure 4b) [14] or any geometrical shape [8] circularly polarized microstrip antenna if needed.

\section{ANTENNA DESIGN AND SIMULATION RESULTS}

A microstrip patch with a single-point feed usually radiates linear polarization. If circular polarization is needed, it will be required for two orthogonal patch modes with the same amplitude and with $90^{\circ}$ phase difference to be induced. This can be accomplished by slightly perturbing a patch at appropriate locations with respect to the feed [15]. Conventionally this is done by truncating triangular portions across the diagonal of the patch (as shown in Figure 5a).

As previously stated, two novel single feed circularly polarized (CP) microstrip antennas are developed for this research. The first technique that is used to design a unique circular polarized microstrip antenna is done by truncating circular sectors across the diagonal of a square antenna patch (as illustrated in Figure $5 b$ ). The second technique that is used to fulfill the purpose of designing unique circular polarized microstrip antenna is done by extending circular sectors across the diagonal of a square antenna patch (as presented in Figure 5c). 


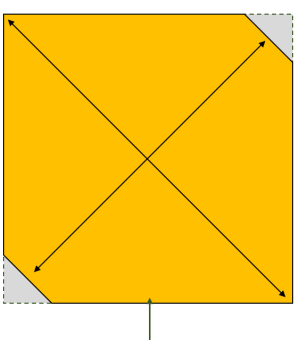

(a) Conventional triangular truncation technique

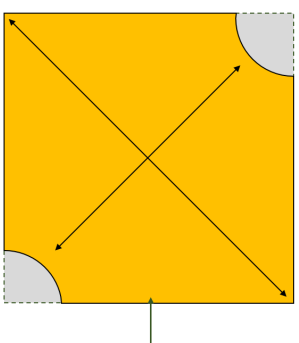

(b) New circular sector truncation technique

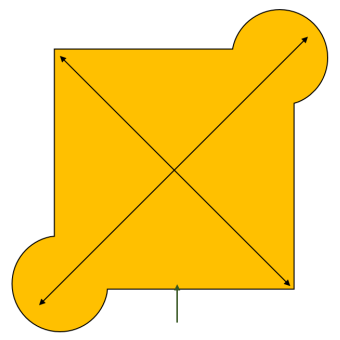

(c) New circular sector extension technique

Figure 5. Conventional and novel techniques to achieve circular polarization with single-point feed

Advanced Design System (ADS) is considered as one of the leading electronic design automation (EDA) software for RF, microwave and high-speed digital applications. This software package is distributed by Keysight Technologies (formerly Agilent's Electronic Measurement). ADS presents an integrated design environment to engineers who need to develop RF electronic products such as microstrip antennas, radar systems, etc. Advanced Design System (ADS) is exclusively used in this research to design and simulate the microstrip antennas.

At first, a Linearly Polarized $2.45 \mathrm{GHz}$ square microstrip antenna is simulated. The resultant parameters of this antenna are used to compare and explain the newly developed Circular Polarized antennas. Some considered values of design parameters which are kept constant for designing all the antennas are presented in the Table 1.

Table 1. Considered design parameters of the proposed antennas

\begin{tabular}{lc}
\hline Design Parameters & Values \\
\hline Dielectric Material & Teflon \\
Dielectric Constant & 2.0 \\
Tan $\delta$ & 0.000194 \\
Thickness of Substrate & $0.8 \mathrm{~mm}$ \\
Thickness of Conductor (Copper) & $0.018 \mathrm{~mm}$ \\
Port Impedance & $50 \Omega$ \\
\hline
\end{tabular}

Figure 6 shows the top view and side view of a $2.45 \mathrm{GHz}$ square microstrip antenna with linear polarization. This antenna is fed by a direct feed microstrip transmission line. The dimensions are also marked in the Figure 6.

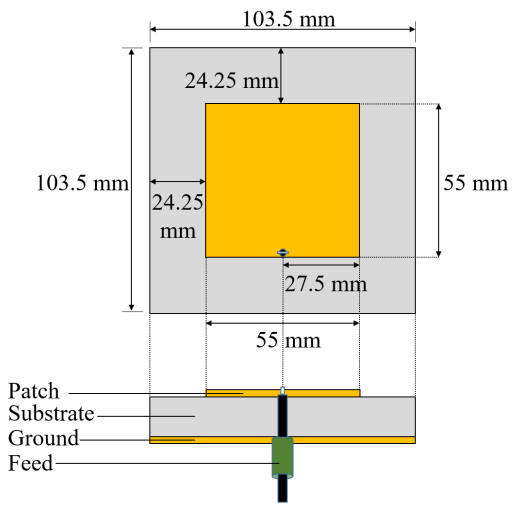

Figure 6. The $2.45 \mathrm{GHz}$ square Microstrip antenna with linear polarization

The return loss, $\mathrm{S}(1,1)$ simulation result of this antenna is shown in Figure 7a. This result indicates that the return loss is lowest $(\approx-40.6 \mathrm{~dB})$ at near of the operating frequency of $2.449 \approx 2.45 \mathrm{GHz}$. Figure $7 \mathrm{~b}$ 
illustrates the Return loss, $\mathrm{S}(1,1)$ on Smith Chart. This graph reveals that the antenna is linearly polarized as there is no visible cusp (sharp fall and immediate rise) on the curve.

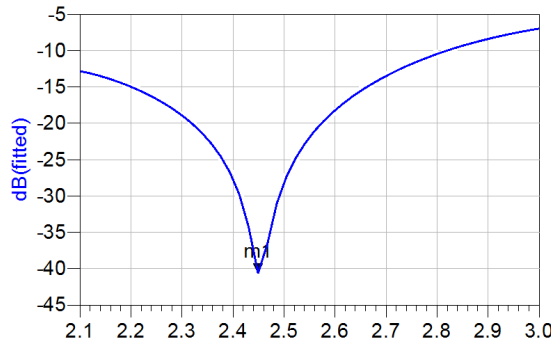

(a) Return loss, $\mathrm{S}(1,1)$ of the linear polarized square microstrip antenna

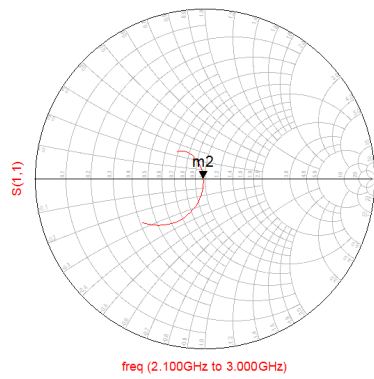

(b) Return loss, $\mathrm{S}(1,1)$ of the linear polarized square microstrip antenna on Smith Chart

Figure 7. S(1,1) parameter of the linear polarized square microstrip antenna

To achieve circular polarization with a single feed the square antenna patch of Figure 6 is modified by slightly perturbing it at suitable locations. Figure 8 shows the top view and side view of the newly designed Crescent-Cut (i.e., circular sector truncated) square microstrip antenna with circular polarization. This antenna is fed by coaxial feeding technique. The dimensions are also marked in the Figure 8.

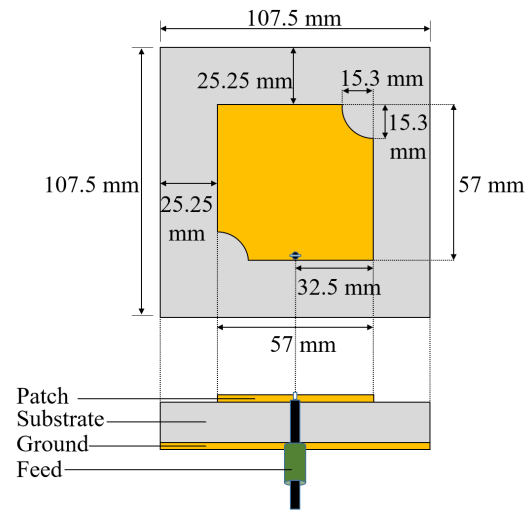

Figure 8 . The crescent-cut microstrip antenna with circular polarization

The return loss, $S(1,1)$ simulation result of this antenna is shown in Figure 9a. This result indicates that the return loss is lowest $(\approx-19.3 \mathrm{~dB})$ at near of the operating frequency of $2.422 \approx 2.42 \mathrm{GHz}$.

To achieve circular polarization this singly fed microstrip antenna works on the principle of detuning degenerate modes of the symmetrical square patch by perturbation of diagonal segments. Thus, the field of a single-point fed patch can be resolved into two orthogonal degenerate modes. Proper perturbation segments will detune the frequency response of any one mode such that, at the operating frequency, it is the equal amplitude and $90^{\circ}$ phase difference with respect to the other mode. Hence, the two modes satisfy the essential conditions for $\mathrm{CP}$ radiation. The detail technical description of this operation is given in [8].

Figure 9b illustrates the Return loss, $\mathrm{S}(1,1)$ on Smith Chart. This graph reveals that the antenna is circularly polarized as there is a cusp (sharp fall and immediate rise) near of the operating frequency on the curve. Note the difference between the curves of Figure $7 \mathrm{~b}$ (Linear Polarization) and Figure 9b (Circular Polarization). The sudden dip in the curve of Figure $9 \mathrm{~b}$ indicates that two orthogonal degenerate modes have formed, and they are resonating in the aforementioned operating frequency. Hence, it confirms the $\mathrm{CP}$ radiation of the antenna. 
Next, the design of the circularly polarized extended corner square microstrip antenna will be discussed. Here, circular polarization is also obtained by a small perturbation of the square antenna patch of Figure 6. Figure 10 shows the top view and side view of the circular sector extended square microstrip antenna with CP. This antenna is fed by coaxial feeding technique. The dimensions are also marked in the Figure 10.

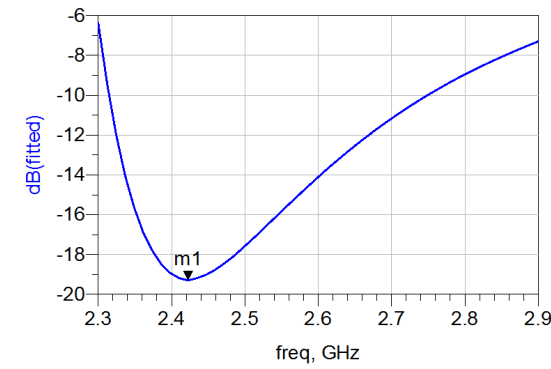

(a) Return loss, $\mathrm{S}(1,1)$ of the circular sector truncated square microstrip antenna with circular polarization

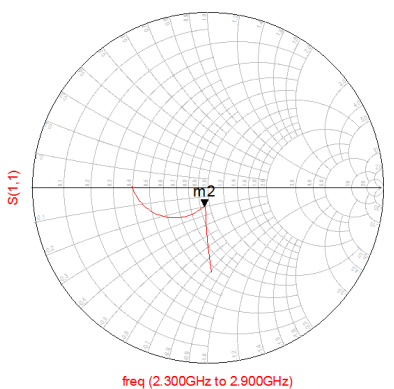

(b) Return loss, $\mathrm{S}(1,1)$ of the circular sector truncated square microstrip antenna with circular polarization on Smith Chart

Figure 9. S(1,1) parameter of the Crescent-Cut circularly polarized antenna

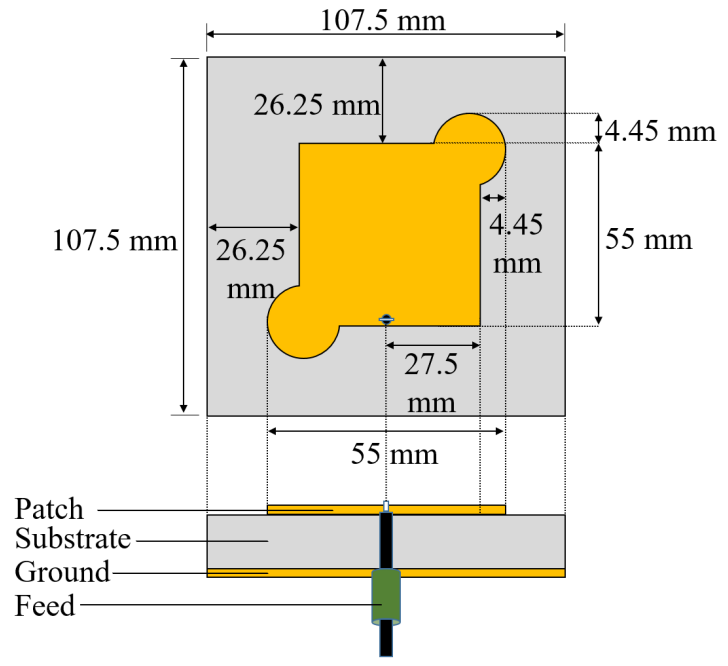

Figure 10. The extended corner microstrip antenna with circular polarization

The return loss, $S(1,1)$ simulation result of this antenna is shown in Figure 11a. This result indicates that the return loss is lowest $(\approx-29.3 \mathrm{~dB})$ at near of the operating frequency of $2.429 \approx 2.43 \mathrm{GHz}$.

To achieve circular polarization this singly fed microstrip antenna works on the principle of detuning degenerate modes of the symmetrical square patch by perturbation of diagonal segments. Thus, the field of a single-point fed patch can be resolved into two orthogonal degenerate modes. Proper perturbation segments will detune the frequency response of any one mode such that, at the operating frequency, it is the equal amplitude and $90^{\circ}$ phase difference with respect to the other mode. Hence, the two modes satisfy the essential conditions for CP radiation. The detail technical description of this operation can be found in [8].

Figure 11b illustrates the Return loss, $\mathrm{S}(1,1)$ on Smith Chart. This graph reveals that the antenna is circularly polarized as there is a cusp (sharp fall and immediate rise) near of the operating frequency on the curve. Note the difference between the curves of Figure 7b (Linear Polarization) and Figure 11b (Circular Polarization). The sudden dip in the curve of Figure $11 \mathrm{~b}$ indicates that two orthogonal degenerate modes 
have formed, and they are resonating in the aforementioned operating frequency. Therefore, it verifies the $\mathrm{CP}$ radiation of the extended corner antenna.

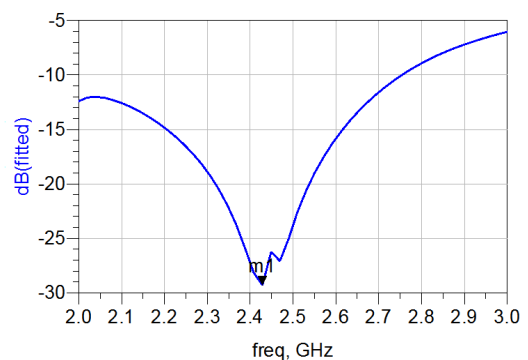

(a) Return loss, $\mathrm{S}(1,1)$ of the circular sector extended square microstrip antenna with circular polarization

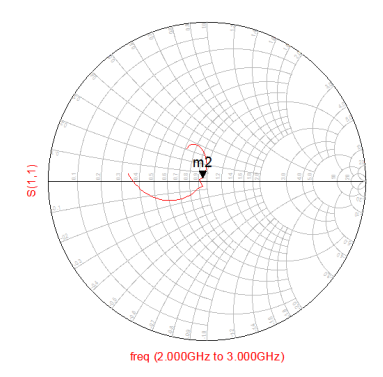

(b) Return loss, $\mathrm{S}(1,1)$ of the circular sector extended square microstrip antenna with circular polarization on Smith Chart

Figure 11. S(1,1) parameter of the extended corner circularly polarized antenna

\section{CONCLUSION}

Two new single feed circularly polarized microstrip antennas at ISM frequency band have been introduced in this paper. Both of the proposed antennas have high gain. At operating frequency, the extended corner antenna has a gain of $5.226 \mathrm{dBi}$, and the gain of the crescent-cut antenna is $5.293 \mathrm{dBi}$. These antennas have been designed and optimized by using Advanced Design System (ADS). In future, these two antenna concepts can be combined to obtain superior performance. Also, unsymmetrical slots inside the radiating patches may be introduced. As these antennas are tuned at ISM band, they are suitable for wireless biotelemetry applications.

\section{REFERENCES}

[1] N. F. Güler and E. D. Übeyli, "Theory and applications of biotelemetry," Journal of Medical Systems, vol. 26, no. 2, pp. 159-178, 2002.

[2] H. R. Khaleel, H. M. Al-Rizzo, D. G. Rucker, and T. A. Elwi, "Wearable yagi microstrip antenna for telemedicine applications," in Radio and Wireless Symposium (RWS), 2010 IEEE. IEEE, 2010, pp. 280-283.

[3] C. H. Doan and D. G. Bach, "Design and fabrication of rectifying antenna circuit for wireless power transmission system operating at ism band," International Journal of Electrical and Computer Engineering (IJECE), vol. 6, no. 4, p. 1522, 2016.

[4] I. Zahraoui, A. Errkik, M. Abounaima, A. Tajmouati, L. Abdellaoui, and M. Latrach, "A new planar multiband antenna for gps, ism and wimax applications," International Journal of Electrical and Computer Engineering (IJECE), vol. 7, no. 4, pp. 2018-2026, 2017.

[5] Spectrum Management Committee, "National Frequency Allocation Plan (NFAP)," 2010.

[6] C. L. Jackson, "The genesis of unlicensed wireless policy," info, vol. 11, no. 5, 2009.

[7] S. Samal, S. Dwari, A. Dutta, and S. P. Reddy, "A microstrip patch antenna for biomedical applications at 2.45 ghz," in Computers and Devices for Communication (CODEC), 2012 5th International Conference on. IEEE, 2012, pp. 1-4.

[8] K.-L. Wong, Compact and broadband microstrip antennas. John Wiley \& Sons, 2004.

[9] H. J. Visser, Array and phased array antenna basics. John Wiley \& Sons, 2006.

[10] C. A. Ballanis, Antenna theory analysis and design. John Wiley \& Sons, 1997.

[11] S. S. Yang, K.-F. Lee, A. A. Kishk, and K.-M. Luk, "Design and study of wideband single feed circularly polarized microstrip antennas," Progress In Electromagnetics Research, vol. 80, pp. 45-61, 2008.

[12] K.-F. Tong and T.-P. Wong, "Circularly polarized u-slot antenna," IEEE Transactions on antennas and propagation, vol. 55, no. 8, pp. 2382-2385, 2007.

[13] H. Iwasaki, "A circularly polarized small-size microstrip antenna with a cross slot," IEEE transactions on antennas and propagation, vol. 44, no. 10, pp. 1399-1401, 1996. 
[14] J.-H. Lu, C.-L. Tang, and K.-L. Wong, "Single-feed slotted equilateral-triangular microstrip antenna for circular polarization," IEEE Transactions on antennas and propagation, vol. 47, no. 7, pp. 1174-1178, 1999.

[15] Q. D. Hossain, M. A. Rahman, and M. A. Hossain, "Dual orthogonal feed circular polarization array antenna using microstrip slot feed network," International Journal of Electrical and Computer Engineering (IJECE), vol. 5, no. 5, 2015.

\section{BIOGRAPHIES OF AUTHORS}
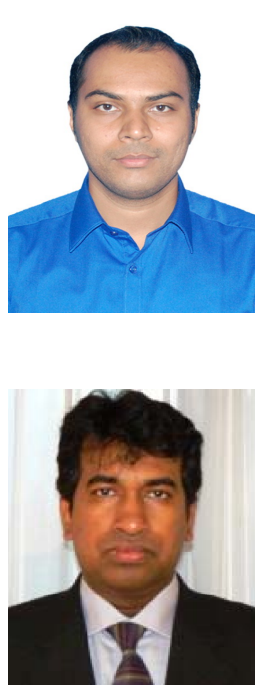

Quazi Delwar Hossain (MIEEE) received his B.Sc. degree in electrical and electronic engineering from Chittagong University of Engineering and Technology (CUET), Chittagong, Bangladesh, in 2001. He obtained Master of Engineering degree in semiconductor electronics and integration sciences from Hiroshima University, Hiroshima, Japan, in 2007; and Ph.D. degree in microelectronics from the University of Trento, Trento, Italy, in 2010. During his Ph.D. program, he also spent a period with the SOI Group, Bruno Kessler Foundation, Trento, Italy as a Postgraduate Researcher. From 2001, he was with CUET as a faculty member. Presently he is working as a Professor and Head at the Department of Electrical and Electronic Engineering, CUET. His research interests include image sensors, antenna design and related readout circuit simulation and experimental characterization of semiconductor devices, sensors and photovoltaic cells.

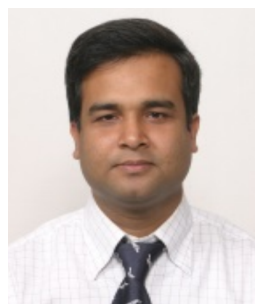

Md. Azad Hossain (MIEEE) received the B.Sc. degree in Electrical and Electronic Engineering from Rajshahi University of Engineering and Technology (RUET), Rajshahi, Bangladesh, in 2004. He got Master of Engineering degree in EEE from Saga University, Saga, Japan, in 2010; and the Ph.D. degree in Science and Advanced Technology, in 2013 from the same Institute. From 2013, he was with the Department of Electronics and Telecommunication Engineering at Chittagong University of Engineering and Technology (CUET) as a faculty member. Presently he is working as an Associate Professor at CUET. His research interests include Microwave antenna design and related readout circuit simulation and experimental characterization.

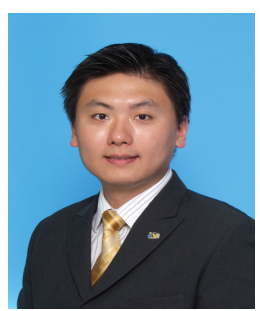

Ray Chak Chung Cheung (MIEEE) received the B.Eng. (Hons) and M.Phil. degrees in computer engineering and computer science \& engineering from the Chinese University of Hong Kong (CUHK) in 1999 and 2001 respectively, and the DIC and Ph.D. degree in computing from Imperial College London (IC) in 2007. After completing his Ph.D. study, he received the Hong Kong Croucher Foundation Fellowship and moved to Los Angeles, in the Electrical Engineering department at UCLA, where he spent two years with Image Communication Lab for continuing his research work. He is currently an Associate Professor in the Department of Electronic Engineering at the City University of Hong Kong and with Digital Systems Lab. His current research interests include cryptographic hardware designs and design exploration of System-onChip (SoC) designs and embedded system designs. 